\title{
Introspection créatrice et comédie humaine. Proust, Balzac et Bergson ${ }^{1}$
}

\author{
Thomas Carrier-Lafleur \\ Université Laval et Université Paul-Valéry (Montpellier III)
}

Des femmes passent dans la rue, différentes de celles d'autrefois, puisque ce sont des Renoir, ces Renoir où nous refusions jadis de voir des femmes. Les voitures aussi sont des Renoir, et l'eau et le ciel [...]. Marcel Proust, Le Côté de Guermantes

Dans un fragment célèbre du Contre Sainte-Beuve (dorénavant $C S B)$, Proust se livre à une étude de Balzac et de son œuvre. « La

\footnotetext{
${ }^{1}$ Cet article a été écrit avec le soutien financier du Conseil de recherches en sciences humaines du Canada (Programme de bourse d'études supérieures du Canada J. Armand Bombardier).
} 
vulgarité de ses sentiments est si grande que la vie n'a pu l'élever » (CSB, p. 187), est-il dit en ouverture. La rhétorique de Proust est la suivante : faire de Balzac un personnage de Balzac. Et pour quel motif ? Celui de l'ambition sociale. Comme dans les plus cinglants morceaux de LaComédie humaine, sont mélangées «les plus basses ambitions» et «l'élite de la société ». On cherche «le grand moyen de domination». La réussite sociale semble être le seul but recherché. L'absolu est social. Balzac, qui proclame haut et fort vouloir concurrencer l'état civil, devient l'arroseur arrosé, comme en témoigne cette correspondance, savamment relevée par Proust, où il parle avec sa sœur Laure de son mariage prochain avec Mme Hanska. Du grand amour de sa vie, qu'il aime depuis plus de quinze ans, il écrit : «Le cœur, l'esprit, l'ambition ne veulent pas en moi autre chose que ce que je poursuis depuis seize ans; si ce bonheur immense m'échappe, je n'ai plus besoin de rien. Il ne faut pas croire que j'aime le luxe. J'aime le luxe de la rue Fortunée avec tous ses accompagnements: une belle fermme, bien née, dans l'aisance et avec les plus belles relations » (CSB, p. 187-188; l'auteur souligne). Le bonheur serait essentiellement mondain et la plume est rythmée par un désir de reconnaissance sociale. «Balzac met tout à fait sur le même plan les triomphes de la vie et de la littérature » (CSB, p.189). Si ce n'est pas La Comédie humaine qui le rendra grand aux yeux de la société, ce sera ce mariage. Le sentiment, littéraire ou amoureux, aussi idéal soitil, n'est peut-être qu'un prisme à travers lequel passe l'ambition... Balzac construit sa vie comme il le ferait pour un roman, puisque l'auteur paraît épris du même désir d'ascension sociale que ses personnages les plus notoires, à l'inverse de Proust, qui a dû quitter le monde des salons pour écrire son œuvre. Par ailleurs, le style de Balzac se définirait comme un 
non-style. C'est que le style, pour l'auteur d'À la recherche $d u$ temps perdu, doit être une traduction de notre « moi profond »; comme la couleur pour le peintre, le style est vision, il recompose la réalité. Le réel ne doit pas être représenté tel quel. Cette doxa proustienne, appliquée au microcosme balzacien, n'est pas viable, et pour cause, car le style de Balzac est avant tout une explication. Du coup, il insère plusieurs images, aussi saisissantes que justes, mais qui ne s'harmonisent pas avec la composition générale. Par l'image, tout est donné et tout s'actualise. Le roman balzacien ne peut être qu'au présent, parce que l'auteur « se sert de toutes les idées qui lui viennent à l'esprit, et ne cherche pas à les faire entrer, dissoutes, dans un style où elles s'harmoniseraient et suggéreraient ce qu'il veut dire » (CSB, p. 203). Or, doit-on en conclure que, s'il en est un, le style de Balzac est inorganisé et naïf...

Si la question peut rester ouverte, c'est que Proust n'a pas peur des paradoxes. Après avoir fait l'inventaire de toutes ces « vulgarités » littéraires, il laisse ainsi entendre qu'il faut malgré tout aimer Balzac, « avec un tout petit peu d'ironie qui se mêle à la tendresse » $(C S B$, p. 210), contrairement à un écrivain comme Tolstoï, que nous devons lire de façon quasi dogmatique. En somme, il faut excuser Balzac, car il est à la recherche de l'effet. Sa Comédie humaine tire sa valeur littéraire du retour constant des personnages de roman en roman. Montage saccadé, mais montage tout de même, tel «un rayon détaché du fond de l'œuvre » (CSB, p. 213) qui est toujours susceptible d'illuminer les personnages, les lieux, les sentiments, etc. Ce dispositif, Proust - qui a bien lu et relu Balzac (plus encore que Tolstoï) - le reprendra dans son propre roman. La Recherche est certainement une série d'études analytiques, philosophiques, de mœurs, avec leurs scènes de la vie privée, de 
province, parisienne, de campagne, politique et militaire. Néanmoins, Proust n'est pas Balzac. Sa Comédie humaine n'est pas sociale, mais intérieure. Ce que Proust recherche, c'est, avançons-nous, l'introspection créatrice. La vérité se trouverait non pas dans le réel à l'état brut, mais bien gravée dans le temps intime et secret de notre vie. C'est ce que le présent article tentera de démontrer, à travers une étude comparative du devenir écrivain et du style propres à ces deux fondateurs du roman moderne que sont, à leur manière respective et pour ainsi dire d'une façon complémentaire, Proust et Balzac.

\section{Images médiatrices du devenir écrivain}

Balzac tient une place considérable dans le roman proustien, dépassant ainsi le mélange d'ironie et de tendresse qui caractérisait la critique qu'en a d'abord faite Proust. Auteur fétiche de Charlus, adoré de Swann, lu et commenté par le héros-narrateur, il est également au cœur des arguments littéraires les plus houleux. Dans Sodome et Gomorrhe, tome par excellence de la décadence mondaine et de la peinture sanglante de la société, le narrateur demande au baron ce qu'il préfère dans La Comédie humaine. La réponse de Charlus sera sans équivoque : tout, il préfère tout, aussi bien ce qu'il nomme les «miniatures" (Le Curé de Tours, La Femme abandonnée) que les «grandes fresques» telles les Illusions perdues. Étrangement, le héros proustien n'a jamais lu cet ouvrage, qui, avec sa suite Splendeurs et misères des courtisanes, forme une partie totale de l'œuvre balzacienne, c'est-à-dire une fenêtre privilégiée dont la vue englobe tout l'univers de l'auteur. Cela 
permet à Charlus un rare cri du cœur². Puis l'universitaire Brichot se joint à la conversation, quitte à provoquer un débat. « $[\mathrm{A}] \mathrm{u}$ risque de contrister les âmes en mal de déférence balzacienne, sans prétendre, Dieu me damne! au rôle de gendarme de lettres et dresser procès-verbal pour fautes de grammaire, j'avoue que le copieux improvisateur dont vous me semblez surfaire singulièrement les élucubrations effarantes, m'a toujours paru un scribe insuffisamment méticuleux » $(S G$, p. 438), dit-il pour ouvrir son intervention. Sur Illusions perdues, il ajoute que «ces romans-feuilletons rédigés en pathos, en galimatias double et triple [...], m'ont toujours fait l'effet des mystères de Rocambole, promu par inexplicable faveur à la situation précaire de chef-d'œuvre» (ibid.). La réponse de Charlus à cette offensive est aussi simple qu'incisive : "Vous dites cela parce que vous ne connaissez pas la vie» (ibid.). On trouve l'illustration de cette impression, qui n'est pas seulement un mot d'esprit, un tome en aval, dans La Prisonnière, alors que le narrateur, qui paraît avoir enfin développé son jugement critique sur La Comédie humaine, propose à son amante Albertine une analogie entre l'art de Wagner et celui de Balzac. Balzac, donc, «jetant sur ses ouvrages le regard à la fois d'un étranger et d'un père, [...] s'avisa brusquement en projetant sur eux une illumination rétrospective qu'ils seraient plus beaux réunis en un cycle où

\footnotetext{
2 «Comment! vous ne connaissez pas les Illusions perdues? C'est si beau, le moment où Carlos Herrera demande le nom du château devant lequel passe sa calèche : c'est Rastignac, la demeure du jeune homme qu'il a aimé autrefois. Et l'abbé alors de tomber dans une rêverie que Swann appelait, ce qui était bien spirituel, la Tristesse d'Olympio de la pédérastie. Et la mort de Lucien ! je ne me rappelle plus quel homme de goût avait eu cette réponse, à qui lui demandait quel événement l'avait le plus affligé dans sa vie: "La mort de Lucien de Rubempré dans Splendeurs et Misères". » (SG, p. 437-438)
} 
les mêmes personnages reviendraient et ajouta à son œuvre, en ce raccord, un coup de pinceau, le dernier et le plus sublime " (Pr, p. 150-151). Cette unité, trouvée après coup, est pour le héros, futur homme de lettres, ce qu'il y a de plus réel et de plus beau. La vie, le sens d'une vie, ne fonctionne pas autrement. Ce qu'avait bien vu Charlus.

Balzac aurait certes accepté ces éloges, mais tout porte à croire qu'il y aurait ajouté une nuance. La composition de son œuvre, avant de refléter la vie, épouse d'abord la structure de la société, à travers, comme il le dit dans l'«Avant-propos » (dorénavant «A-P») de LaComédie humaine, une « comparaison entre l'Humanité et l'Animalité » («A-P», p. 7), en ceci que « la Société ressembl[e] à la Nature » («A-P», p. 8). Le concept de la différence est au cœur de ce système, et la composition multiforme, labyrinthique, des romans tente d'en reproduire la forme. Le matériau brut de l'œuvre balzacienne n'est pas tant le réel que les «Espèces sociales » (ibid.) qui y vivent et qui transforment la réalité à leur image. L'ordre social est alors bien plus complexe que l'ordre naturel puisque, dans le premier, le hasard fait rage. Les renvois et les retours des personnages dans les différents morceaux de LaComédie humaine sont là pour souligner et resouligner la logique étonnante de l'état civil que, selon le mot célèbre de Balzac, le romancier doit concurrencer. Or, derrière cet apparent désordre du hasard, se cache une loi. C'est alors l'ambition du romancier que d'en dicter les principes fondateurs. Balzac, avec cette recherche romanesque des lois sociales que nous subissons sans pour autant les percevoir, dit faire plus que l'histoire avec ses « sèches et rebutantes nomenclatures » («AP», p.9). L'idée lui est justement venue par la lecture des romans dits historiques de l'écrivain écossais Scott, car celui-ci 
«imprimait [...] une allure gigantesque à un genre de composition injustement appelé secondaire » («A-P », p. 10). La grande force de Scott est que ses personnages sont des images du présent. Sa faiblesse, toutefois, est de ne pas avoir construit un système cohérent capable d'unir et de dérouler ces images. Le présent, pour être représenté et pour survivre dans un roman, doit être pris en charge par l'art de la liaison. Et c'est bien cela que Balzac, "[f]ondateur incontesté du roman moderne » (Dubois, p. 170), entend pallier. Plus encore que la réalisation d'une peinture sociale, Balzac souhaite en étudier les raisons, les effets et les sens cachés, en un mot, le «moteur social» («A-P», p. 11) avec tous ses principes naturels qui, pour être réels, doivent absolument être pris sur le vif. Alors, suivant ces indications, que pourrait bien être l'image médiatrice de la création et du devenir littéraire chez Balzac, celle qui en dessine le système ? Nul besoin de chercher bien loin, car on la trouvera également chez lui, cette fois dans la préface de Splendeurs et misères des courtisanes (dorénavant $S M C$ ), lorsque l'auteur, toujours lucide quant à sa démarche, dit vouloir «daguerréotyper une société » (p. 50). La métaphore de la photographie, instantanée surtout, voire du cinématographe, aurait sans doute été plus adéquate, mais on ne peut reprocher à Balzac de ne pas avoir pu connaître et expérimenter ces inventions. Or, La Comédie humaine serait le photo-roman du $X^{X}{ }^{e}$ siècle français, non pas son roman-feuilleton. Balzac fait des clichés de la société : les hommes, les femmes et les choses doivent être saisis à chaud, dans leur élément naturel. L'assemblage des daguerréotypes constitue la composition de l'œuvre, comme si l'ordre social posait pour le romancier qui doit en tirer, comme on dit justement en photographie, les lois. Les retours de personnages - unité ultérieure et non factice - 
deviennent la profondeur de champ de l'image romanesque de Balzac et témoignent de l'acuité de sa vision du présent et de la réalité environnante. S'il n'est pas «vrai dans les détails » («A$P »$, p. 15), le roman ne serait rien. Pour reprendre à nouveau les mots de J.Dubois, le projet balzacien exigeait «non seulement un plan mais encore tout un appareil conceptuel et analytique» (p. 176). Le daguerréotype, ancêtre de la photographie que les écrivains naturalistes dans la lignée de Zola feront leur, est cette machine ${ }^{3}$.

De son côté, Proust, même s'il donne aussi une place de choix à la photographie dans son œuvre, d'un point de vue autant narratif qu'esthétique, est pourtant méfiant envers cette passion pour l'image qui prétend représenter la réalité. De Balzac, il conservera la pensée sociale et la beauté de l'agencement. La Recherche, avec ses centaines de personnages, offre une sociologie pratique, étudiant les classes et les mouvements de la société, décortiquant les causes et les effets d'événements cinglants telles l'Affaire Dreyfus et la Grande Guerre, mettant en scène les jeux de coulisses et les passions secrètes des individus et des masses. Néanmoins, la réalité qui intéresse Proust n'est pas celle qui obsédait Balzac. Peut-on imaginer le narrateur de la Recherche, à quelque moment que ce soit de son apprentissage, vouloir concurrencer l'état civil en se faisant secrétaire d'une société qui lui dicterait le seul livre valant la peine d'être écrit? Certainement pas, puisqu'une des haines de Proust est justement celle de «la littérature de

\footnotetext{
${ }^{3}$ En plus de préfigurer l'enregistrement photographique du réel propre à l'univers balzacien, le daguerréotype est aussi à rapprocher d'une typologie, à savoir d'une poétique du type trouvant son embryon imaginaire dans la littérature de mœurs qui a fortement influencé Balzac (tel l'Adolphe de Benjamin Constant).
} 
notations » (TR, p. 201). À l'observation sur le vif de la réalité et des lois du hasard qui la gouvernent, Proust apporte une variante majeure qui est celle du temps. Là où Balzac cherche à juxtaposer l'espace, Proust rend le temps sensible. Par là même, la réalité prend un sens nouveau, qui n'est autre que celui qu'elle doit avoir à nos yeux. Elle est une réalité hétérogène, qui, de façon sérielle, se déroule en nous. La réalité proustienne est d'abord intérieure. «Peu à peu, conservée par la mémoire, c'est la chaîne de toutes ces expressions inexactes où ne reste rien de ce que nous avons réellement éprouvé, qui constitue pour nous notre pensée, notre vie, la réalité » (ibid.), lit-on dans Le Temps retrouvé. On le voit, pareille esthétique ne peut se fonder sur l'observation, aussi minutieuse soit-elle, de l'espace social qui nous entoure. Le fonctionnement est différent : ce n'est pas la mimétique ou même, à l'inverse, la face cachée des choses qui est importante, c'est leur transformation, imperceptible à l'œil nu, un changement qui, pourrions-nous dire, se fait dans l'écoulement de notre durée intérieure, un temps qui n'est certes pas linéaire. Cet art de vivre, manière toute spéciale de comprendre la vie, semble être une réponse du narrateur à Charlus, comme si, ayant progressé dans son parcours d'écrivain, se rapprochant du but qui est l'adoration perpétuelle de l'œuvre d'art, il avait tiré les leçons nécessaires pour émettre une opinion originale, sa propre théorie sur la vie. Du même coup, la réponse va de Proust à Balzac. Cette introspection créatrice, fer de lance disions-nous de la stylistique proustienne, s'échelonne sur bien des degrés qui se déploient dans le récit selon l'avancement de l'apprentissage esthétique du héros. Une des premières manifestations de ce phénomène créateur apparaît lors des moments de lecture du narrateur, encore enfant à Combray. Même jeune, il est doté d'une qualité 
de lecture hors du commun, d'une sensibilité extrême s'approchant d'un sixième sens, si bien qu'

une fois que le romancier nous a mis dans cet état, où comme dans tous les états purement intérieurs, toute émotion est décuplée, où son livre va nous troubler à la façon d'un rêve mais d'un rêve plus clair que ceux que nous avons en dormant et dont le souvenir durera davantage, alors, voici qu'il déchaîne en nous pendant une heure tous les bonheurs et tous les malheurs possibles dont nous mettrions dans la vie des années à connaître quelques-uns $(C S, \mathrm{p} .84)$.

L'analogie avec le rêve, espace-temps idéal où notre vie intérieure s'active pour prendre forme, est essentielle. Elle renvoie aux premières pages du roman, à cet étrange prologue, sorte de fable ou de discours de la méthode, insituable dans la chronologie du roman, énoncée par un «je » inconnu, qui doit bien être le héros-narrateur, mais qu'on ne peut malgré tout identifier. «Un homme qui dort tient en cercle autour de lui le fil des heures, l'ordre des années et des mondes » (CS, p. 5), nous est-il dit d'entrée de jeu. Ce qui frappe, comme l'a remarqué Doubrovsky, et ce qui confère un statut ambigu à ce fragment du roman proustien, «c'est que, dès les premiers mots, dès les premières pages, le destin littéraire de la Recherche était joué » (p. 45). Du moins, si tout n'est pas joué, une certaine impression de totalité se manifeste, comme si l'apprentissage du narrateur, construit en étapes, en déceptions et en réussites, devait progressivement ramener le lecteur à cet état, qui n'est pas sans rappeler celui de grâce. Doubrovsky souligne un autre point majeur, sur lequel on insistera : " [1]a formidable machinerie textuelle est d'emblée opératoire, sous forme miniaturisée. Interférences temporelles, déconstruction des perspectives, et patiente poursuite, inlassable recomposition du moi contre la dispersion de l'être » (p. 46), 
vraiment, tout y est, tout est donné, mais, pour un premier lecteur, tout est encore à venir, car si Proust nous livre d'emblée son secret, il n'a pas pour autant dit son dernier mot. Pourtant, l'image médiatrice du devenir littéraire du narrateur proustien ne sera pas le rêve. Il faut, comme on vient de le voir, et comme c'était le cas pour Balzac, une machine.

Nous avons dit que ce n'était pas la photographie, vision trop directe, pour ne pas dire appauvrie, de la réalité. Le cinématographe, alors, évolution créatrice de la photographie instantanée? Encore moins, puisque, comme le dit Proust, «ce que nous appelons la réalité est un certain rapport entre ces sensations et ces souvenirs qui nous entourent simultanément - rapport que supprime une simple vision cinématographique, laquelle s'éloigne par là d'autant plus du vrai qu'elle prétend se borner à lui » (TR, p. 196). Le vrai, la réalité, il ne faut pas s'y borner. La logique proustienne du roman demande une certaine distance, une réfraction, qui n'est possible que par l'amalgame de nos souvenirs et de nos sensations, c'est-à-dire par la mécanique de notre vie intérieure. Tel un conseil que Proust donne aux romanciers à venir (et plusieurs l'écouteront), il ajoute que ce rapport unique et privilégié, « l'écrivain doit [le] retrouver pour en enchaîner à jamais dans sa phrase les deux termes différents » et les " enferm[er] dans les anneaux nécessaires d'un beau style » (ibid. pour les deux citations). Le film cinématographique (Proust entend par là le cinéma des premiers temps) ne représente que des lieux communs. Au mieux, il y ajoutera quelques détails. Or, la Recherche, même si elle en renferme des milliers, n'est pas construite par des détails et des anecdotes. Ce n'est pas un choix de la part de Proust. Si on en croit le parcours allégorique de son narrateur alter ego, une stylistique originale 
s'est littéralement imposée à lui. Avec elle, l'image médiatrice de son fonctionnement, que l'on décèle dans le passage qui suit la lecture du volume inédit du Journal des Goncourt (où Proust offre un pastiche délirant du style des deux frères écrivains, comme il l'avait déjà fait ailleurs avec "L'affaire Lemoine»). Après la lecture de ce Journal, c'est d'abord la déception qui prime. Les Goncourt et le héros ont fréquenté les mêmes gens et connu le même monde, mais ils en ressortent, pour le moins, avec des impressions différentes. Les Goncourt savent raconter de judicieuses anecdotes, dépeindre le caractère des invités avec intelligence et précision. Tout cela échappe au narrateur. Le passage qui suit, s'il peut se lire comme une confession, doit davantage être compris comme un manifeste: "J'avais beau dîner en ville, je ne voyais pas les convives, parce que, quand je croyais les regarder, je les radiographiais» (TR, p. 25). On a peine à concevoir Balzac avouer qu'il a dépeint l'ensemble de la société française du XIXe siècle alors qu'il ne savait pas voir. Comment aurait-il pu « copi[er] toute la Société, la saisi[r] dans l'immensité de ses agitations » («A-P», p. 14) en étant étranger à l'observation? La réalité que cherche à radiographier Proust, c'est, par exemple, «l'identité du salon Verdurin dans divers lieux et dans divers temps», réalité qui est située "à miprofondeur, au-delà de l'apparence elle-même, dans une zone un peu plus en retrait » (TR, p. 24, pour les deux citations). Qui plus est, cette zone en retrait est double ; elle se manifeste deux fois : d'une part en plongeant de plus en plus à l'intérieur de nous, d'autre part, en radiographiant de plus en plus profondément le monde, les êtres et les choses qui nous entourent, ce qui fait de la radiographie proustienne une radiographie intérieure et temporelle. Les divers lieux et les divers temps du salon Verdurin n'existent nulle part, exception 
faite de la vie intérieure des êtres qui y ont pris part, dont le narrateur, seul écrivain du lot en excluant les Goncourt. On comprend ainsi que l'opposition stylistique entre la Recherche et La Comédie humaine se fait sur deux plans. D'un côté, là où Balzac a un sens aigu de l'observation, Proust tord son intelligence pour regarder à l'intérieur de lui-même. De l'autre côté, le temps balzacien est linéaire ; en d'autres mots, il est une variable indépendante. C'est un temps mathématique; il n'est pas humain. Le temps réellement perdu ne se trouve pas dans la Recherche, mais dans une œuvre comme La Comédie humaine. $\mathrm{Au}$ mieux, Balzac fera resurgir le passé dans un instantané, lorsqu'un personnage est rattrapé par son destin. Mais ce temps-là n'est pas créateur ; il suit son cours et ronge la vie sur son passage. À l'inverse, le temps proustien est circulaire, à commencer par la circularité du roman qui se termine par le bruit de clochette, dernière réminiscence, qui rappelle le départ de Swann à Combray, c'est-à-dire le début de l'œuvre. Le phénomène est semblable pour la description $\mathrm{du}$ salon Verdurin que propose le narrateur, puisqu'il doit puiser dans ses souvenirs et suivre l'écoulement intérieur de la durée pour traverser les apparences du présent qui, trop tourné vers l'avenir, ne peut qu'être mensonger. Proust a su rendre la vie intérieure romanesque, précisément parce que c'est elle qui crée le romanesque. Le temps de notre conscience est un temps créateur. Proust pousse cette idée à l'extrême, en évoquant maintes fois le danger de l'entreprise. C'est le cas du personnage du jaloux, que l'auteur élève presque au niveau de personnage conceptuel. La jalousie proustienne n'est pas tant quelque chose qui s'observe que quelque chose qui se crée: « des incidents aussi sont amenés par la vie intérieure ; [...] les hasards rencontrés dans les réflexions que je faisais seul me 
fournissaient parfois de ces petits fragments de réel qui attirent à eux, à la façon d'un aimant, un peu d'inconnu qui, dès lors, devient douloureux. On a beau vivre sous l'équivalent d'une cloche pneumatique, les associations d'idées, les souvenirs continuent à jouer » $(P r$, p. 18), dit le narrateur, pensant à la possible homosexualité d'Albertine. Notre vie intérieure est un théâtre, une sorte de société secrète qui rivalise elle aussi avec l'état civil. Encore le héros pensant à Albertine, qu'il vient de rencontrer quelques jours plus tôt lors de son premier séjour à Balbec: «j'avais fait chaque jour à son sujet des milliers de réflexions, j'avais poursuivi, avec ce que j'appelais elle, tout un entretien intérieur où je la faisais questionner, répondre, penser, agir, et dans la série indéfinie d'Albertines imaginées qui se succédaient en moi heure par heure, l'Albertine réelle, aperçue sur la plage, ne figurait qu'en tête, comme la "créatrice" d'un rôle, l'étoile, ne paraît, dans une longue série de représentations, que dans les toutes premières » $(J F$, p. 421422). Tout semble alors indiquer que Proust, devant le génie et la force de Balzac, dans un curieux rapport amour-haine, ait tenté de dépasser le maître en proposant non pas une, mais deux comédies humaines : d'abord extérieure et spatiale, puis intérieure et temporelle, la première servant de moteur à la seconde. Pour clarifier cette opposition, on ira voir du côté de la philosophie bergsonienne, avec laquelle notre champ lexical flirte depuis un certain moment.

\section{Bergson, le comique et le tragique}

D'une part, nous avons donc Balzac à l'affût des espèces sociales, un pied dans le présent et l'autre dans l'avenir rapproché pour déceler les tendances, les lois et les types de 
l'état civil. Autrement dit, c'est un homme d'action. Sans penser, il agit, il réagit aux mouvements sociaux les plus divers dans le dessein d'en brosser le tableau le plus exact, tout en détail, le plus fidèle à la réalité qu'il voit. Projet ambitieux, qui, sans arrêt, nous oblige à jouer, jouer dans les méandres du social et du politique pour apprendre à en tirer les ficelles. D'autre part, il y a Proust et sa recherche intérieure, introspective, du temps perdu qu'il faut retrouver en nous. Certes, c'est une sortie du monde (dans la biographie de Proust, elle arrivera vers 1909), mais pour mieux y retourner, par une forme de sociabilité nouvelle qui est celle du livre. Le rêve et les souvenirs à l'état pur sont des vecteurs d'une vérité qui ne peut se trouver qu'avec le temps. Ce pour quoi on avancera que le personnage type - entendons par là le personnage conceptuel — de Balzac doit être l'automate, alors que celui de Proust est le rêveur. Deux extrêmes, deux façons de vivre, mais également deux manières d'écrire et de créer. Matière et mémoire (dorénavant $M M$ ) nous donne en quelque sorte le mode d'emploi de ces deux personnages. D'abord le rêveur proustien, à savoir «un être humain qui rêverait son existence au lieu de la vivre [et qui] tiendrait [...] sous son regard, à tout moment, la multitude infinie des détails de son histoire passée » (MM, p. 172). Ensuite l'automate balzacien, « celui, au contraire, qui répudierait cette mémoire avec tout ce qu'elle engendre jouerait sans cesse son existence au lieu de se la représenter véritablement : automate conscient, il suivrait la pente des habitudes utiles qui prolongent l'excitation en réaction appropriée » (ibid.).

Le rêve et les souvenirs offrent ainsi une vie nouvelle à celui qui en fait son modus operandi. Dans la Recherche, rêver sa vie est un processus tout à fait opératoire et qui n'est pas dénoué d'affects intenses: «J'avais les larmes aux yeux comme 
ceux qui, seuls dans leur chambre, imaginant selon les détours capricieux de leur rêverie la mort d'un être qu'ils aiment, se représentent si minutieusement la douleur qu'ils auraient, qu'ils finissent par l'éprouver » ( $\operatorname{Pr}$ p. 341), dit par exemple le narrateur. Le rêve est un moteur romanesque qui fonctionne avec le rhizome des souvenirs, tout autant qu'il les fait fonctionner. De ce va-et-vient intérieur, d'une vitesse parfois prodigieuse (il suffit de penser à la réminiscence de la petite madeleine rappelant Combray autant qu'elle le crée), naît une idée d'art. Encore, l'idée d'un art original, d'une étrange forme de réalisme intérieur, par là plus vrai que l'extérieur puisqu'il est vécu et revécu une infinité de fois. Le passage qui suit, l'une des phrases les plus longues de la Recherche (qu'il faut citer en entier pour, justement, en comprendre tout le mouvement), exprime parfaitement ce que Proust nomme parfois « l'alchimie » de la création littéraire. Le narrateur, fasciné par Orianne, la duchesse de Guermantes, qu'il commence à fréquenter, tente, lorsqu'il n'est pas avec elle, de se la représenter mentalement, grâce à ses souvenirs et à partir des idées romanesques sur les femmes aimées qu'il avait déjà forgées, que ce soit avec Gilberte ou Albertine :

Auprès de ces idées, le souvenir de Mme de Guermantes à l'Opéra était bien peu de chose, une petite étoile à côté de la longue queue de sa comète flamboyante; de plus je connaissais très bien ces idées longtemps avant de connaître Mme de Guermantes; le souvenir, lui, au contraire, je le possédais imparfaitement; il m'échappait par moments ; ce fut pendant les heures où, de flottant en moi au même titre que les images d'autres femmes jolies, il passa peu à peu à une association unique et définitive - exclusive de toute autre image féminine - avec mes idées romanesques si antérieures à lui, ce fut pendant ces quelques heures où je me le rappelais le mieux que j'aurais dû m'aviser de savoir exactement quel il était; 
mais, je ne savais pas alors l'importance qu'il allait prendre pour moi ; il était doux seulement comme un premier rendezvous de Mme de Guermantes en moi-même, il était la première esquisse, la seule vraie, la seule faite d'après la vie, la seule qui fût réellement Mme de Guermantes; durant les quelques heures où j'eus le bonheur de le détenir sans savoir faire attention à lui, il devait être bien charmant pourtant, ce souvenir, puisque c'est toujours à lui, librement encore, à ce moment-là, sans hâte sans fatigue, sans rien de nécessaire ni d'anxieux, que mes idées d'amour revenaient ; ensuite, au fur et à mesure que ces idées le fixèrent plus définitivement, il acquit d'elles une plus grande force, mais devint lui-même plus vague ; bientôt je ne sus plus le retrouver; et dans mes rêveries, je le déformais sans doute complètement, car, chaque fois que je voyais Mme de Guermantes, je constatais un écart, d'ailleurs toujours différent, entre ce que j'avais imaginé et ce que je voyais. $(C G$, p. 54$)$

Ici, Proust ne tente aucunement de daguerréotyper sa vie intérieure. C'est précisément l'inverse. La conscience, le rapport entre nos sensations et nos souvenirs, on ne peut les rendre visibles ni les représenter. Les détails de notre vie intérieure sont infinis ; le romancier réaliste, même le plus hardi, ne peut en rendre compte objectivement. Mais si, au contraire, l'écrivain accepte le branle de la mémoire, les multiples directions de sa boussole intérieure, il comprendra que c'est dans la logique spéciale de ce mouvement qu'il sera à même de trouver une esthétique originale qui, en sortant de lui-même, lui présentera le monde sous un jour nouveau. L'universel, pour le rêveur, est d'abord individuel. Une minute de son passé contient tout le temps de sa vie, voire de la vie de tous les hommes. Chez Proust, c'est-à-dire chez le rêveur, l'ordre civil ne peut être qu'intérieur. Pour l'automate, à savoir l'homme d'action qui refuse les zigzags de la mémoire involontaire, l'ordre civil est tout ce qu'il y a de plus concret. C'est ce dans quoi il évolue et performe 
quotidiennement. Dans l'action, les deux pieds dans le temps linéaire de la société, il joue avec ce qu'il nomme les faits pour établir des certitudes, ou, dit autrement, il «amass[e] tant de faits [pour] les peindre comme ils sont» («A-P», p. 16). Tellement ancré dans l'action, l'écrivain automate n'hésite pas à se donner une fonction sociale majeure, « une décision [...] sur les choses humaines » («A-P», p. 12), qui « le rend égal et peutêtre supérieur à l'homme d'État » (ibid.). Balzac aimait à citer une phrase de Louis de Bonald, lorsque le philosophe avance $\mathrm{qu}^{\prime}$ « $[\mathrm{u}] \mathrm{n}$ écrivain doit avoir en morale et en politique des opinions arrêtées, il doit se regarder comme un instituteur des hommes; car les hommes n'ont pas besoin de maîtres pour douter»(ibid.). De la clarté et encore de la clarté. L'idée balzacienne doit être claire, arrêtée, fixe et précise comme une photographie, à l'inverse de la radiographie proustienne qui se donne la tâche, non pas moins ardue, d'explorer le réseau infini et circulaire des souvenirs et des sensations. En somme, deux conceptions radicalement opposées de l'art romanesque et de la création littéraire, et, pour parler avec Bergson, «deux états extrêmes, l'un d'une mémoire toute contemplative qui n'appréhende que le singulier dans sa vision, l'autre d'une mémoire toute motrice qui imprime la marque de la généralité à son action » (MM, p. 173). Pour comprendre la différence, pas seulement de degré, mais bien de nature, entre la stylistique mémorielle de Balzac et celle de Proust, on tentera maintenant d'éclaircir le rôle de la comédie dans l'œuvre de l'écrivain automate, tout en proposant la tragédie comme théâtre intérieur dans lequel le rêveur crée à son tour. On quittera aussi Matière et mémoire pour le produit suivant de la pensée bergsonienne, Le Rire (dorénavant, $R$ ). 
Publié en 1900, quatre ans après Matière et mémoire, Le Rire peut être lu à la fois comme un livre de philosophie, d'analyse littéraire et sociologique, dont les grands sujets, au sens médical du terme, sont d'abord et surtout l'automate impulsif, tourné vers l'action, et ensuite le rêveur, plongé dans ses souvenirs. Tous deux souffrent d'une pathologie, d'un certain déséquilibre dans la balance entre l'esprit et le corps, la mémoire et la matière. Ce sont des pathologies parce que la vie ne nous présente que très rarement de tels individus, aussi portés vers l'extrême. Or, Bergson, dans Le Rire, se tourne comme jamais dans son œuvre vers la littérature, car c'est le privilège et la force du romancier, de l'homme de théâtre ou du poète d'offrir et de créer des spécimens semblables. C'est aussi pourquoi nous disions plus haut que l'automate et le rêveur sont deux personnages conceptuels. À travers eux se déploie une idée. Le comique, qui relève surtout de l'automate, est alors un dérèglement de la machine humaine, une distraction face à la vie qui mérite d'être soulignée, pour ne pas dire châtiée. Il y a aussi un comique propre au rêveur, lui aussi un grand distrait, mais ce qui est vraiment intéressant avec lui, ce que nous essaierons de montrer, c'est comment la comédie fait place à la tragédie, phénomène que l'on ne remarque pas chez l'automate impulsif, étranger à l'introspection. C'est que le rêveur, on l'a vu rapidement avec le héros proustien, tend à se détacher du monde. Il s'écarte donc progressivement du rire, car, comme le dit Bergson, « [p] our comprendre le rire, il faut le replacer dans son milieu naturel, qui est la société; il faut surtout en déterminer la fonction utile, qui est une fonction sociale » $(R$, p. 6). Pas de rire sans une société qui le manie, qui s'en sert. Non seulement le rire est une fonction, il a une utilité, mais sa fonction est sociale, civile. Balzac n'est pas loin, et du coup 
s'éclaire le dessein de sa Comédie humaine. En effet, cette proposition bergsonienne ne manquera pas d'être comprise par un lecteur de l'œuvre de Balzac: «[l]e comique naîtra [...] quand des hommes réunis en groupe dirigeront tous leur attention sur un d'entre eux, faisant taire leur sensibilité et exerçant leur seule intelligence» (ibid.). La comédie, chez Bergson comme chez Balzac, pour le philosophe et pour le romancier, est affaire de morale. Dans La Comédie humaine, c'est Balzac, avec sa force herculéenne, qui incarne l'ensemble de la société dirigeant son attention, et finalement sa plume, vers un de ses membres, le personnage fautif, qui devra être jugé et par là remis à l'ordre, l'ordre civil, bien entendu. La finalité de la société et, cela va de soi, celle de l'écrivain du social, est de travailler pour son perfectionnement, d'amender ses fautes et ses péchés ${ }^{4}$. Le comique, rire de la société, a quelque chose à enseigner à l'individu ou au groupe qui fait fausse route, qui se détourne de l'ordre et de la vie. Balzac insiste souvent sur la valeur professorale du roman tel qu'il l'entend et le pratique, parce que « [l]'enseignement [...] est [...] le grand principe d'existence pour les peuples, le seul moyen de diminuer la somme du mal et d'augmenter la somme du bien dans toute Société » ("A-P», p. 12-13). Les personnages de Balzac seraient ainsi coupables du même vice que leur auteur, le vice de l'automatisme. L'auteur, ne serait-ce que dans ses romans (on connaît tous les problèmes de Balzac avec sa propre vie sociale, qui paraît être une série de malentendus et d'occasions manquées), a toutefois une carte cachée, puisque

\footnotetext{
4 «L'homme n'est ni bon ni méchant, il naît avec des instincts et des aptitudes ; la Société, loin de le dépraver, comme l'a prétendu Rousseau, le perfectionne, le rend meilleur» («A-P», p. 12), dit Balzac dans l'ouverture $a$ posteriori de sa Comédie humaine.
} 
c'est lui qui mène le jeu. Il est alors le meneur des marionnettes; les personnages sont des instruments, des pantins, représentant chacun une espèce ou un mixte d'espèces. Ce qui fait sourire Proust devant l'œuvre immense de Balzac ne peut être que cet automatisme respectivement subi et partagé entre l'auteur et ses créations, ainsi que la « vulgarité » du style de Balzac qui reflète la morale qu'il tente d'inculquer à ses personnages, mais qui est aussi celle qui frappe l'auteur dans sa biographie. Pour Balzac, on voit qu'il y a autant de vices qu'il y a de groupes dans la société, car c'est «la Famille et non l'Individu [qui est] le véritable élément social » («A-P », p. 13), et chaque fragment de sa Comédie s'attache à une famille particulière: les nobles, les bourgeois, les paysans, les journalistes, les hommes politiques, les acteurs, les poètes, les avocats, la police, les voleurs, les courtisanes, etc. Chaque famille a son heure de gloire, ou, plutôt, son jugement dernier, dans l'œuvre balzacienne. Et Balzac de tenir parole lorsqu'il affirme haut et fort que " [l] es actions blâmables, les fautes, les crimes, depuis les plus légers jusqu'aux plus graves, y trouvent toujours leur punition humaine ou divine, éclatante ou secrète » («A-P», p. 15). C'est que le roman, tel que Balzac le conçoit pour ne pas dire qu'il l'invente, doit «tendre vers le beau idéal ». Contrairement à l'histoire, qui manque de style et de morale, «le roman doit être le monde meilleur » (ibid. ; l'auteur souligne), dit-il en reprenant les mots de Mme Necker (l'épistolière Suzanne Curchod). Plus encore, le roman permet de croire aux "progrès de l'homme sur lui-même » ( $A-P$ », p. 16), car l'homme n'est pas une créature finie. La tâche de La Comédie humaine est écrasante : dans une fiction, dépeindre et daguerréotyper les quelque 2000 ou 3000 personnages qui forment une époque pour en saisir les différents types, les 
diverses familles, avec le plus de détails possible, pour ensuite en cerner les vices particuliers, puis les punir, et dans un deuxième souffle ramener la société sur la voie du beau et du bon. Dans ce livre ostensiblement comique, dit Balzac, se trouve «l'application des grands principes d'ordre, de politique, de moralité » («A-P », p. 19). À bien lire, il n'est donc pas exagéré de concevoir avec Bergson (qui paraît ici renvoyer, sans le dire, au projet romanesque balzacien) que le rire est « une espèce de geste social. Par la crainte qu'il inspire, il réprime les excentricités » $(R$, p. 15 ; l'auteur souligne). C'est aussi par le rire que Balzac peut dialoguer avec Bergson ou encore avec des sociologues tels Émile Durkheim ou Gabriel Tarde, puisque, écrit le philosophe, «le rire ne relève [...] pas de l'esthétique pure, puisqu'il poursuit [...] un but utile de perfectionnement général. Il a quelque chose d'esthétique cependant puisque le comique naît au moment précis où la société et la personne, délivrées du souci de leur conservation, commencent à se traiter elles-mêmes comme des œuvres d'art » $(R$, p. 15-16). Auteur et personnage, individus et groupes, société et roman, tout est mélangé. Tous sont aux aguets, automates conscients et comiques, ils se surveillent, se réprimandent, se châtient, mais toujours dans cette volonté progressiste et évolutionniste qui est propre au rire. Le comique est équivoque : il n'appartient pas tout à fait à l'art, et pas tout à fait à la vie. N'est-ce pas là le plus beau compliment que l'on pourrait faire à Balzac et à sa grande machine réaliste?

«Quel est l'objet de l'art ? Si la réalité venait frapper directement nos sens et notre conscience, si nous pouvions entrer en communication immédiate avec les choses et avec nous-mêmes, [...] l'art serait inutile, ou plutôt que nous serions tous artistes, car notre âme vibrerait alors continuellement à 
l'unisson de la nature. Nos yeux, aidés de notre mémoire, découperaient dans l'espace et fixeraient dans le temps des tableaux inimitables. [...] Nous entendrions chanter au fond de nos âmes, comme une musique quelquefois gaie, plus souvent plaintive, toujours originale, la mélodie ininterrompue de notre vie intérieure. » ( $R, \mathrm{p} .115)$ Suivant de près ces lignes, qui sont parmi les plus belles de toute l'œuvre de Bergson, on trouve la fameuse métaphore du voile qui s'interpose entre la nature et nous, plus encore, entre nous et notre conscience. La vie de tous les jours est trop centrée sur l'action, sur l'utilité. De la réalité vraie, nous ne percevons qu'un symbole, une simplification pratique. Ce qui échappe à la quotidienneté, c'est l'individualité. «[J] usque dans notre propre individu, l'individualité nous échappe » $(R, \mathrm{p} .118)$, ajoute Bergson, cette fois dans un élan proustien. Ne lit-on pas dans Le Temps retrouvé que «[1]a grandeur de l'art véritable, [c'est] de retrouver, de ressaisir, de nous faire connaître cette réalité loin de laquelle nous vivons, de laquelle nous nous écartons de plus en plus au fur et à mesure que prend plus d'épaisseur et d'imperméabilité la connaissance conventionnelle que nous lui substituons, cette réalité que nous risquerions fort de mourir sans avoir connue, et qui est tout simplement notre vie» (TR, p. 202). On comprend pourquoi la comédie, dont la fonction première est sociale, serait à mi-chemin entre l'art et la vie, puisqu'elle ne permet pas l'introspection créatrice ; elle ne lève pas le voile de l'habitude ; elle cherche plutôt à en expliquer le motif qui y est brodé. Déchirer le rideau de notre conscience, c'est donc le propre du drame : « il va chercher et amène à la pleine lumière, [...] une réalité profonde qui nous est voilée, souvent dans notre intérêt même, par les nécessités de la vie » $(R, \mathrm{p}$. 121). Le drame révélera les contradictions de la société, plutôt que de la 
photographier ou encore d'en décréter les lois. L'individualité d'un Hamlet - ou encore celle du héros-narrateur proustien est certaine. Toutefois, c'est dans ce qu'elle a de plus singulier qu'elle pourra devenir universelle et acceptée comme telle. C'est la logique particulière du drame, mise en lumière par la philosophie bergsonienne de l'art, et revendiquée par Proust, son contemporain : « [p]lus grande est l'œuvre et plus profonde la vérité entrevue, plus l'effet pourra s'en faire attendre, mais plus aussi cet effet tendra à devenir universel » $(R, \mathrm{p} .125)$. L'universalité est la ligne d'arrivée de l'entreprise dramatique d'introspection, alors qu'elle est le point de départ de la comédie humaine et sociale. La généralité est ce qui fait fonctionner la comédie. Des scènes de la vie privée, politique, de la province à Paris, elle dénote, recoupe et assemble les éléments communs pour les présenter au lecteur, lui-même devenu, à l'instar de l'auteur comique moraliste, juge et policier. Les retours des personnages dans LaComédie humaine indiquent bien que nous ne pouvons pas échapper à la société, que l'on peut changer de classe, de profession ou de passion : nous sommes toujours susceptibles d'être pris dans les mailles de ce filet qu'est l'ordre civil, filet de plus en plus vaste selon l'acuité visuelle du romancier tout-puissant. De là aussi tous les jeux de reflets entre les divers héros comiques, entourés de personnages secondaires, qui en représentent des copies simplifiées, accentuant la lisibilité du réel. Le narrateur de la Recherche n'a pas d'alter ego, sinon Proust. Certains se risqueront avec Swann, mais Swann est l'alter ego de ce que le narrateur n'est pas, de la partie comique de son être qu'il sut mettre de côté pour avancer dans son apprentissage artistique, qui est, on le voit bien, dramatique et introspectif. Le héros proustien, d'abord un personnage comique, lorsqu'il se 
promène dans les cercles de la mondanité puis dans ceux de l'amour, tend progressivement à devenir un personnage tragique. La Recherche raconte le passage de la comédie à la tragédie, qui, pourrait-on suggérer, marque aussi le passage du $\mathrm{XIX}^{\mathrm{e}}$ au $\mathrm{XX}^{\mathrm{e}}$ siècle, de l'observation extérieure à l'observation intérieure. La première actualise les lois qu'elle croit déceler dans l'ordre civil; la seconde, selon la belle expression de Bergson, «saisit le virtuel dans le réel» $(R$, p. 129). Aussi l'observation comique ne peut-elle que porter sur les autres, car nous ne sommes comiques qu'en société, et le rire ne se comprend et ne se représente que par « des rapprochements de données éparses, par une comparaison entre des cas analogues dont on exprime la quintessence, enfin par un travail d'abstraction et de généralisation semblable à celui que le physicien opère sur les faits pour en dégager des lois » $(R$, p. 130). Or, Balzac, au début de son "Avant-propos», ne manque pas d'évoquer Frédéric Cuvier et Étienne Geoffroy Saint-Hilaire. Dans La Comédie humaine, la comparaison entre l'humanité et l'animalité fait graviter l'entreprise romanesque autour du noyau des sciences exactes. Au contraire, une entreprise comme celle de Proust passe du comique à une certaine forme de tragique. D'abord, on dégage les lois de la mondanité, de la guerre, de l'amour et de la politique ; ensuite, et c'est là le plus important, il faut lire en soi-même pour y trouver une réalité plus profonde. Le passage va du comique au tragique, des lois à la lecture de soi. La dimension sociale du comique a besoin de la généralité ; elle se situe entre l'art et la vie, alors que la tragédie et l'introspection sont de l'art pur. De la transition entre la comédie humaine et l'introspection créatrice, on proposera maintenant une étude textuelle, d'abord 
de Balzac et ensuite de Proust, pour juger de la valeur des concepts qui ont été quelque peu éclaircis et comparés.

\section{Splendeurs et misères... de la vanité}

Pour enchaîner, on se permettra de rappeler un autre passage du Rire bergsonien, celui où il est question du rapport entre la vanité, le rire et le social, rapport que le philosophe, par la suite, laisse de côté : "Une étude complète des illusions de la vanité, et du ridicule qui s'y attache, éclairerait d'un jour singulier la théorie du rire. [...] On verrait comment la vanité, qui est un produit naturel de la vie sociale, gêne cependant la société, de même que certains poisons légers sécrétés continuellement par notre organisme l'intoxiqueraient à la longue si d'autres sécrétions n'en neutralisaient l'effet » $(R, \mathrm{p} .133)$. Or, la vanité est précisément ce qui caractérise le personnage de Lucien dans Illusions perdues (dorénavant IP). Ce défaut de caractère, qui s'aiguise en se manifestant sous diverses formes au cours du récit, le fait chavirer du rêve à l'automatisme. De poète, il devient journaliste. L'âme de Lucien semble être disputée entre deux régimes antagonistes qui, de personnage tragique, le transforment progressivement en personnage comique, au sens bergsonien du terme. Splendeurs et misères des courtisanes mettra le point final à cette métamorphose, faisant de Lucien, à son retour à Paris, la «chose» de Jacques Collin. À la lettre, Lucien est maintenant une courtisane; il fait partie du même monde que les filles de la rue.

Proust résume ainsi le roman de Balzac: «Lucien de Rubempré venant à Paris s'est rendu compte que Mme de Bargeton était ridicule et provinciale, que les journalistes 
étaient fourbes, que la vie était difficile. Illusions toutes particulières, toutes contingentes, dont la perte peut l'acculer au désespoir et qui donnent une puissante marque de réalité au livre, mais qui font rabattre un peu de la poésie philosophique du titre » $(C S B$, p. 200). La comédie est bel et bien entre l'art et la vie, comme les illusions balzaciennes sont avant tout sociales. Lucien ne pourra être ni poète ni écrivain, ayant pourtant écrit un recueil de poèmes (ses Marguerites) et un roman (L'Archer de Charles $(X)$, non pas parce qu'il manque d'inspiration ou de sujet, mais bien parce qu'il n'a pas d'éditeur, parce que les libraires sont des crapules, parce que la poésie ne se vend plus, etc. Balzac déplore le sort de la littérature, sans pour autant insister sur des raisons esthétiques. Des grands romans et des poèmes sublimes peuvent encore s'écrire, mais on a arrêté de les lire. Ce qui est décrié est plutôt l'industrialisation de la littérature au XIXe siècle. Pour Balzac, le grand coupable de cette transformation commerciale est la presse. Dans Illusions perdues, le journal est une machine privilégiée du rire et de sa sanction sociale. Ainsi, « [les] journalistes ne pouvaient pas [...] échapper à la juridiction de la comédie » (IP, p. 50), étant sous l'emprise des "mœurs horriblement comiques de la Presse » (p. 51), que Balzac est un des premiers à mettre en scène. Pour l'auteur de La Comédie humaine, la presse est toute-puissante ; elle sert l'ordre social et humilie ceux qui ne veulent ou ne peuvent se plier aux nouvelles règles civiles, tels les poètes, êtres distraits et lunaires, plongés dans leurs souvenirs et dans leurs impressions intimes au détriment de la vie sociale et de l'action. Si Balzac a fait de Lucien, pseudo-poète, un journaliste accompli, voire prodigieux, entouré de ses acolytes, Lousteau, Nathan, Blondet, figures du double qui hantent Lucien en même temps qu'elles expliquent son caractère en devenir, c'est pour 
mieux saisir sur le vif les «commencements de la maladie» provoqués par les mœurs terribles du journal, «ce cancer qui dévorera peut-être le pays » (IP, p. 52 pour les deux citations). La vanité de Lucien le pousse à réussir, mais, dans La Comédie humaine, on ne peut réussir que socialement. « Le pauvre grand homme de province» (IP, p. 454) va quitter les hauteurs de sa vie spirituelle et tomber dans les bas-fonds de la société pour rejoindre " les damnés de l'enfer parisien qui se battent à coup d'encrier » (IP, p. 53). Allégorie de la poésie et de la beauté, il deviendra progressivement une victime de la société qui tentera de le réformer. Paris lui coupera les ailes et le privera des hauteurs du rêve. Il quittera l'introspection créatrice pour l'observation mécanique d'une société cannibalesque qui veut l'engloutir. La vie, dorée par la couleur du rêve, deviendra aussi noire que les caractères imprimés des presses infernales. «Les pensées dominantes et la vie intérieure» (IP, p. 79) seront elles-mêmes dominées par la geste sociale et cruelle du comique. Si Balzac peut déployer avec force cette imagerie de la perdition comique des grands idéaux littéraires et d'une existence vouée à la contemplation passive mais créatrice, c'est que Lucien est coupable d'un défaut originel, ayant mordu « à la pomme du luxe aristocratique et de la gloire» (IP, p. 107). Toute illusion sera perdue et ainsi seront éclairées une à une « [l]es vanités de ce poète » (IP, p. 159).

À Paris, Lucien n'est plus dans son élément: le poète balzacien n'est pas un homme social, mais un reclus de la société, ce que l'ordre civil lui fera chèrement payer en le prenant dans son jeu machinal. À Angoulême, un personnage tel Châtelet, rival de Lucien pour l'amour de Mme de Bargeton, paraît pour le moins bête. Lucien pourra avoir le dessus sur lui, l'amour chez Balzac étant une sorte de tauromachie sociale. 
Mais, dans la capitale, les rôles changent : Lucien est puni pour son manque d'aptitudes sociales alors que «Châtelet, élégant et à son aise comme un acteur qui retrouve les planches de son théâtre, regagnait en deux jours tout le terrain qu'il avait perdu en six mois » (IP, p. 193). La ville est dotée d'une vitesse sociale. Il faut arriver à suivre la parade mondaine, ce que Lucien ne peut faire avec ses idéaux. Pour concurrencer Châtelet, il devra jouer sur le même terrain que lui, qui est celui de l'impulsif et non plus du rêveur. Tout à coup lui apparaît « le monde des superfluités nécessaires » (IP, p. 197). Des espérances du ciel, il tombe dans les réalités de la boue qui ne se transformera pas en or. La rencontre avec d'Arthez et les autres membres du Cénacle changera quelque peu la donne. Dans le désert parisien, le Cénacle est une oasis miraculeuse, une sphère tout en hauteur où « personne ne pensait aux réalités de la vie que pour en tirer d'amicales plaisanteries» (IP, p. 242), car elle est peuplée «d'intelligences presque divines » (IP, p. 245). Ses neuf membres, d'Arthez en premier lieu, sont prêts à l'accueillir à bras ouverts, puisqu'ils voient en lui une immense source de talent. Puis, en discutant avec Lucien et en le fréquentant, ils se rendront compte que sa vanité le rend inapte au travail littéraire sérieux. Un furieux désir d'ambition mondaine lui fait dépenser toute son énergie à des tâches pourtant futiles. C'est dans ce monde, c'est-à-dire c'est dans le monde, que Lucien choisit de réussir, et pas dans le dur labeur de l'homme de lettres, qui, pour contourner les pièges que lui tend l'industrialisation de la littérature, doit redoubler d'ardeur pour que l'inspiration puisse se déployer et ainsi faire une œuvre. Michel Chrestien, membre du Cénacle qui porte bien son nom, parlant de sa vanité, annonce à Lucien sous une forme prophétique ce qu'il perçoit de son futur : « Il y a chez toi [...] un 
esprit diabolique avec lequel tu justifieras à tes propres yeux les choses les plus contraires à nos principes. " La suite est primordiale : «au lieu d'être un sophiste d'idées, tu seras un sophiste d'action.» (IP, p. 247, pour les deux citations) Né poète, mais poète ambitieux et vaniteux, Lucien devra devenir autre, passer de l'idée à l'action, de la pensée à l'automatisme, quitter les hauteurs pour les bas-fonds, le paradis intellectuel pour l'enfer du journalisme, etc. Les conseils des membres du Cénacle n'auront, et encore pas pour longtemps, que peu de prise sur Lucien, qui mélange tout et juge d'Arthez et ses frères spirituels à partir de leur position sociale inférieure, voire médiocre, alors que c'est précisément contre la société que ces derniers l'ont prévenu. Lucien, selon les mots de Balzac, souhaite plutôt se « jeter » dans le journalisme. Au sens figuré, mais aussi au sens propre. Il en développe les qualités, dont la première est «la soudaineté de la pensée » (IP, p. 249). Une pensée, comme le laisse entendre Bergson dans Matière et mémoire pour caractériser la logique propre à l'automate, tournée vers l'avenir rapproché, une pensée pratique, sans spiritualité, une pensée qui frise la non-pensée. L'auteur de La Comédie humaine prête alors sa voix à Fulgence, autre membre du Cénacle, lorsqu'il déclare solennellement à Lucien que « [l]e journalisme est un enfer, un abîme d'iniquités, de mensonges, de trahisons, que l'on ne peut traverser et d'où l'on ne peut sortir pur, que protégé comme Dante par le divin laurier de Virgile » (ibid.). Or, Lucien ne trouvera son protecteur qu'après avoir sombré encore plus bas que les journalistes, dans l'Enfer des enfers, expulsé de Paris comme d'Angoulême, sur le bord de la rivière qu'il a choisie pour lit de mort. Et, comme Lucien n'est pas Dante, il est évident que Collin, alias Vautrin, Carlos Herrera et Trompe-la-mort, ne vaut pas Virgile... 
«Est comique tout arrangement d'actes et d'événements qui nous donne, insérées l'une dans l'autre, l'illusion de la vie et la sensation nette d'un agencement mécanique », écrit Bergson (R, p. 53 ; l'auteur souligne). Cette phrase résume et explique le parcours de Lucien de la province à la capitale, le rapport entre les illusions et les pertes, le moteur du désir et le fonctionnement de la vanité. Un temps, Lucien croit vivre et pense réussir. Ses premiers articles ont un succès fou ; il gagne de l'argent, fréquente une jolie actrice de 18 ans ; il est respecté des hommes qui le jugent comme un rival et admiré des femmes qui le voient comme une conquête. L'image utilisée par Balzac est aussi frappante qu'amusante : «Lucien avait un pied dans le lit de Coralie, et l'autre dans la glu du Journal» (IP, p. 318). Il semble chez lui dans « ces mauvais lieux de la pensée appelés journaux » (IP, p. 322), allant même jusqu'à qualifier les journalistes d'êtres extraordinaires, "supérieurs aux hommes graves et sérieux du Cénacle » (IP, p. 323). Toutefois, cette vie de luxe et de luxure dans laquelle Lucien est pour le moment heureux sera «la pente du précipice où il devait tomber» (ibid.). Il croyait jouer avec le monde, mais c'est la société qui tire les ficelles. Lucien, dont les splendeurs finiront toutes en misères, est un pantin à ficelles, image par excellence de la comédie. Pour Bergson, un personnage comique est un pantin lorsqu'il « croit parler et agir librement, [et] où [il] conserve par conséquent l'essentiel de la vie, alors qu'envisagé d'un certain côté il apparaît comme un simple jouet entre les mains d'un autre qui s'en amuse » (R, p. 59). De la mécanique plaquée sur du vivant, voilà ce qui attend Lucien dans ses tribulations parisiennes, où le rêve fait place au pur automatisme, au comique de situation et de caractère. " $[\mathrm{T}] \mathrm{u}$ pourras être un grand écrivain, mais tu ne seras jamais qu'un petit farceur » (IP, 
p. 336), scande à Lucien un des membres du Cénacle, peiné de voir son ancien ami pris dans cette toile d'araignée qu'est l'état civil dans La Comédie humaine.

«[E]ntraîné par un courant invincible dans un tourbillon de plaisirs et de travaux faciles [...], Lucien vécut au jour le jour » (IP, p. 388-389). Mais il ne se doute pas que le tourbillon deviendra en moins d'un an un maelström. Ses amis et collègues journalistes deviendront ses ennemis ; ses ouvrages, qui étaient promis à un brillant succès (grâce à ses relations), seront calomniés puis oubliés ; il perdra son appartement ; la carrière théâtrale de son amante Coralie sera en chute libre si bien qu'elle tombera gravement malade pour mourir quelques mois plus tard. La dernière scène parisienne d'Illusions perdues, un des plus beaux coups de pinceau de Balzac, montre Lucien, par terre, à côté du cadavre encore chaud de Coralie, en train d'écrire quelques chansons grivoises qui lui donneront l'argent nécessaire pour l'enterrement de la jeune actrice. Même dans la mort, il faut jouer le jeu... Rastignac, lui aussi d'Angoulême, résumera ainsi le sort de Lucien à Ève, la sœur du feu poète : "Votre frère est un aiglon que les premiers rayons du luxe et de la gloire ont aveuglé. Quand un aigle tombe, qui peut savoir au fond de quel précipice il s'arrêtera?» (IP, p. 479) On a les précipices que l'on mérite. Celui de Lucien serait ironiquement une rivière, rappelant par le débit de ses flots le doux rythme de la conscience et de la vie intérieure. C'est au bord de cette rivière où il comptait terminer ses jours que Lucien fera la rencontre la plus déterminante de sa vie: Jacques Collin, sous les traits de l'abbé espagnol Carlos Herrera, personnage clé de La Comédie humaine, doté d'une puissance mentale et physique surhumaine, parfait meneur d'hommes, d'une volonté de fer, mais malheureusement au service du mal. Collin est un 
personnage en lutte contre la société. Génie du crime, sultan du bagne, il concurrence l'état civil en formant sa propre société secrète, composée de malfrats qui lui obéiront au doigt et à l'œil. Collin fera donc comprendre ses torts à Lucien, le premier étant de s'être pris pour un maître, d'avoir tenté de contrôler tout le monde, alors qu'il n'était encore qu'un élève. La société s'est vengée, et elle l'a puni. Dans « ce grand théâtre appelé le monde » (IP, p. 593), théâtre comique cela va de soi, Lucien ne pouvait d'emblée tenir le rôle principal, mais il ne pouvait se contenter d'un rôle secondaire. Ce qui manquait à Lucien, dit Collin, c'est un maître, quelque esprit de taille pouvant lui montrer à avancer masqué, à se jouer de tout sans jamais être joué, pouvant lui apprendre à tirer les ficelles. Avec Collin, Lucien est en présence de l'automate supérieur. "Je vous ai pêché, je vous ai rendu la vie, et vous m'appartenez comme la créature est au créateur » (IP, p. 596), dit le premier au second. C'est un « pacte d'homme à démon », ajoute Balzac (ibid.). C'est, toujours dans un sens bergsonien, une scène fortement comique, à savoir que «le comique est ce côté de la personne par lequel elle ressemble à une chose, cet aspect des événements humains qui imite, par sa raideur d'un genre tout particulier, le mécanisme pur et simple, l'automatisme, enfin le mouvement sans la vie » $(R, \mathrm{p} .66-67)$. C'est ce que sera Lucien, comme il le dit, non sans gêne, dans une lettre destinée à sa sœur: " $\mathrm{Au}$ lieu de me tuer, j'ai vendu ma vie. Je ne m'appartiens plus, je suis plus que le secrétaire d'un diplomate espagnol, je suis sa créature. Je recommence une existence terrible. Peut-être aurait-il mieux valu me noyer » (IP, p. 615). Collin, dans Splendeurs et misères des courtisanes, sera l'ordre sous lequel Lucien agira, achevant sa transformation de rêveur en impulsif. 
Lucien sera en effet une « jolie machine » (SMC, p. 107) à laquelle Collin pourra dire: «j'ai tout conçu de toi [...], j'ai converti ton manque de touche au jeu de la vie en une finesse de joueur habile» $(S M C$, p. 112). De retour à Paris, il a abandonné toute pensée de gloire littéraire, alors que ses Marguerites ont finalement pu éclore et que son roman a été republié ; à ce sujet, il dira seulement que " [c']est un succès posthume » (SMC, p. 125). Lucien est devenu le double de Collin, un second lui-même. Plutôt que d'escalader lentement l'échelle sociale, il a changé de voie et explore maintenant les manières obscures pour contourner et rouler la société et l'ordre civil. Il est un «mort-civil» (SMC, p. 144). Collin-Herrera, double faussaire, pense pour Lucien ; plus encore, il lui évite de penser, faisant de lui une marionnette des bas-fonds et un objet de révolte. Dans les niveaux inférieurs et secrets de la société, se jouera d'ailleurs une lutte comique, où seront convoqués forçats, espions, filles de joie, voleurs et même violeurs. Le romancier veut faire parler leur histoire, muette jusque-là. Collin est le roi de cette racaille. Or, par ses machinations, il y entraîne Lucien, bien loin maintenant des hautes sphères du rêve et de la poésie. Un vaudeville visant à renflouer le portefeuille de Lucien et, si possible, lui conférer une position sociale de choix sera bientôt mis en place par le faux abbé espagnol. Une fois l'apprentissage de l'automate conscient terminé, ce dernier devra faire payer la société qui l'a fait souffrir. Mais l'état civil n'a pas dit son dernier mot: Collin et Lucien seront emprisonnés, pour ensuite se perdre dans la mécanique de la justice que Balzac ne manque pas de décrire scrupuleusement à son lecteur dans une volonté toute professorale. Illusions perdues et Splendeurs et misères des courtisanes racontent donc la « chute icarienne » (SMC, p. 400) 
de Lucien Chardon de Rubempré, qui avait tout pour être un poète et un rêveur, et même quelque chose en trop, c'est-à-dire la vanité, qui a fait de lui un personnage de comédie, puni et sanctionné par la société parisienne, ramené à l'ordre, si bien qu'il terminera sa route dans une cellule, pendu avec sa cravate, signe que la mondanité l'aura finalement étouffé. Le suicide de Lucien sera la «terminaison d'une aliénation mentale » (SMC, p. 483), résultat d'un trop grand manque d'attention face à la vie ; celle-ci s'est vengée, par le biais de l'ordre civil. À l'heure de son dernier souffle, en écrivant son testament, Lucien paraît comprendre la leçon comique que Balzac tente d'inculquer à ses personnages et qui est un leitmotiv de La Comédie humaine : il faut redonner à la société5. Un dernier coup de plume sera nécessaire. Une lettre adressée à Collin, "grandiose statue du mal et de la corruption» (SMC, p. 486) qui, s'il avait choisi la voie du bien, aurait été " plus que Ximenès, plus que Richelieu » (ibid.).

Proust critiquera cette lettre en disant que « Lucien parle trop comme Balzac » (CSB, p. 198). L'analogie est bien réelle. On peut en effet voir dans le parcours de Lucien, du poète romantique à l'automate comique, une sorte de réfraction de la propre carrière littéraire de Balzac (les poèmes qui composent les Marguerites ont pour la plupart été écrits par l'auteur de

\footnotetext{
${ }^{5}$ Ainsi, l'ancien poète « lègue aux hospices la somme nécessaire à l'achat d'une inscription de rentes de trente mille francs en cinq pour cent. Les intérêts annuels seront employés, pour chaque semestre, à la délivrance des prisonniers pour dettes, dont les créances s'élèveront au maximum à deux mille francs » $(S M C$, p. 483). Ce modèle sera aussi respecté pour la dernière incarnation de Vautrin, où Jacques Collin, décidant de jouer à peu près francjeu, sera engagé par la police, rejoignant les forces de l'ordre en abandonnant sa lutte contre la société. Il sera "médecin des âmes » (SMC, p. 654) et devra «dompt[er] les fous» (SMC, p.655). On le voit, le roman balzacien est un instrument qui vise le bien pour atteindre le meilleur des mondes possibles.
} 
La Comédie humaine dans sa jeunesse). Il le dit d'ailleurs assez clairement dans son "Avant-propos», lorsqu'il mentionne le rêve qui se transforme et qui tombe de son ciel fantastique pour devenir réalité. Le dispositif du retour des personnages (découvert en 1835 avec Le Père Goriot), une fois compris et assumé par Balzac, le fera passer d'auteur romantique, qui écrit sous l'influence de la muse, à un auteur comique, polygraphe social, à mi-chemin entre l'art et la vie. Son inspiration n'est pas intérieure, mais bien extérieure, le romancier devenant une sorte de corps sensible, plaque photographique où viendront s'imprimer la lumière et les reflets de toute la société. La vanité de Balzac, comme celle de Lucien, est mondaine.

\section{Proust, à la recherche de l'absolu littéraire}

Le narrateur proustien est, comme Lucien, un jeune homme de province, doté de certains talents littéraires, qui s'installera à Paris. Il profitera d'une curieuse ascension sociale qui l'amènera au cœur du cercle de l'aristocratie du faubourg SaintGermain en général et des Guermantes en particulier. Il écrira aussi pour les journaux, connaîtra les horreurs de l'amour, fréquentera des actrices et des filles de basse condition. En Charlus, qui offrit au héros-narrateur de devenir son " protégé » ( $C G$, p. 539), la Recherche compte également son Jacques Collin. Contrairement à Lucien, le narrateur refusera la proposition quasi pédérastique du maître, ce qui fait dire à Charlus, raccompagnant le héros chez lui au clair de lune, la réplique suivante: "Mon rôle est terminé, monsieur; j'y ajoute simplement ces quelques paroles. Un autre vous offrira peutêtre un jour sa sympathie comme j'ai fait. Que l'exemple actuel vous serve d'enseignement. Ne le négligez pas. Une sympathie 
est toujours précieuse » (CG, p.547-548). Or, le narrateur proustien n'aurait besoin de la sympathie de personne. En continuant malgré tout à les fréquenter, il perdra progressivement son intérêt pour les Guermantes et autres illustres représentants de l'aristocratie, d'abord perçus comme des êtres mythologiques ${ }^{6}$. La mondanité, dans le roman proustien, est une lamentable chimère qui tend à s'effacer, comme le montre l'ouverture du faubourg Saint-Germain après la Première Guerre mondiale, ou, mieux encore, la montée phénoménale du salon Verdurin. Toutefois, on ne peut que se demander ce qu'aurait été la vie du narrateur s'il avait accepté l'offre de Charlus, comme le fera plus tard Morel (un autre reflet de Lucien). Aurait-il pu écrire ? Se serait-il contenté d'articles dans les journaux? Aurait-il trouvé le sujet de son roman? On peut certes en douter... Mais on comprend surtout que, en refusant la protection de Charlus et en discréditant le monde des salons, le héros refuse de jouer le jeu du comique. Le narrateur de la Recherche, d'abord un personnage de comédie, se dirigera lentement mais sûrement vers la tragédie, c'est-àdire vers l'art pur. Certes, il faut sortir du monde, mais non sans en avoir tiré une leçon. Tout l'apprentissage est là. Proust nous apprend que même le comique le plus strict peut devenir tragique, à savoir qu'il délaissera sa fonction punitive portée sur les groupes sociaux et se fera introspection créatrice à partir de soi-même.

\footnotetext{
${ }^{6}$ Ce type de commentaires, innombrables dans le Côté de Guermantes, le montrent bien : «Je regardais sur lui avec envie ces traits caractéristiques des Guermantes, de cette race restée si particulière au milieu du monde, où elle ne se perd pas et où elle reste isolée dans sa gloire divinement ornithologique, car elle semble issue, aux âges de la mythologie, de l'union d'une déesse et d'un oiseau » $(C G$, p. 73$)$.
} 
Le journal, pour insister sur ce point, ne tient pas le même rôle dans la Recherche que dans La Comédie humaine. En fait, il s'agit de deux systèmes extrêmement différents : le premier, comique, est l'induction de lois; le second, plus tragique, est la déduction de vérités individuelles. Chez Balzac, le journal est l'allégorie des bas-fonds de la société, l'arène dans laquelle le pouvoir se dispute : on y traque les hommes, morts ou vivants. Chez Proust, bien qu'il soit également un incroyable vecteur de mondanité (les aristocrates, comme les bourgeois d'ailleurs, lisent le journal, ils s'en servent et tentent d'y bien paraître), le journal est spécialement, ne serait-ce que pour le héros, un symbole de la communion des esprits et du culte de la lecture, véritable «pain spirituel » $(A D$, p. 149). La publication de son article dans Le Figaro confirme au héros que sa vocation sera littéraire. Il ne pourra pas être autre chose qu'écrivain. Encore faut-il savoir quoi écrire, car un article, malgré la conversion quasi religieuse qu'il provoque, ne fait que souligner la portée de la littérature, il n'est pas une œuvre d'art. Or, Vincent Descombes a tout à fait raison de nuancer le fameux résumé que Genette donne de la Recherche. Ce n'est pas tant que Marcel (le narrateur) devient écrivain, mais plutôt que Marcel devient un grand écrivain. Le récit que propose Proust, à travers l'apprentissage littéraire de son héros, n'est pas un récit classique. L'histoire, aussi bien les faits racontés que le contexte social qui les supporte, paraît bien secondaire en comparaison avec la forme. C'est elle qui est la véritable originalité de l'œuvre, qui transforme la petite histoire en grand récit. «Le sujet de la Recherche [...], ce pourrait être : Illusions perdues. Un début dans la vie, une Éducation sentimentale, etc. Ensuite, il faut transposer l'histoire banale en histoire exceptionnelle. Ce qui arrive au personnage principal est commun [...]. Mais 
l'histoire sera racontée comme le héros la vit, c'est-à-dire comme une aventure incomparable» (Descombes, p. 170). En effet, " [1]a véritable aventure racontée dans le récit n'est plus sociale ou mondaine, mais intellectuelle » (p. 171).

L'aventure intellectuelle se déploie à travers l'image médiatrice de la radiographie temporelle ou, dit autrement, par l'introspection créatrice. Notre conscience épouse ce que Bergson nomme la durée vraie. Toute vérité est celle du temps, à savoir que toute vérité est intérieure et individuelle. C'est une lutte contre l'habitus, il faut se désocialiser, dit Proust de manière convaincante. Il faut chercher sous la matière, sous l'expérience, dépasser l'état civil, sans quelque prétention de vouloir le concurrencer. "Ce travail qu'avaient fait notre amour-propre, notre passion, notre esprit d'imitation, notre intelligence abstraite, nos habitudes, c'est ce travail que l'art défera, c'est la marche en sens contraire, le retour aux profondeurs où ce qui a existé réellement gît inconnu de nous, qu'il nous fera suivre » (TR, p. 203). La séquence du «Bal de têtes ", dernier moment mondain du roman, qui suit de près la révélation esthétique du héros, confirme qu'il a bien appris son étrange leçon. Les êtres qui composent cette matinée, représentants du Tout-Paris, ne valent plus pour l'espèce sociale qu'ils représentent. Il ne reste rien non plus de la mythologie mondaine qui avait obnubilé le héros. C'est une scène purement temporelle dans laquelle la mondanité est intérieure :

Les parties blanches de barbes jusque-là entièrement noires rendaient mélancolique le paysage humain de cette matinée, comme les premières feuilles jaunes des arbres alors qu'on croyait encore pouvoir compter sur un long été, et qu'avant d'avoir commencé d'en profiter on voit que c'est déjà 
l'automne. Alors moi qui depuis mon enfance vivais au jour le jour, ayant reçu d'ailleurs de moi-même et des autres une impression définitive, je m'aperçus pour la première fois, d'après les métamorphoses qui s'étaient produites dans tous ces gens, du temps qui avait passé pour eux, ce qui me bouleversa par la révélation qu'il avait passé aussi pour moi. (TR, p. 233)

Ici s'explique également la métaphore que le narrateur donne pour illustrer sa démarche littéraire et la spécificité de son ouvrage, celle des verres grossissants par lesquels l'auteur et le lecteur pourront lire en eux-mêmes. C'est finalement la différence la plus exemplaire entre le projet balzacien et l'entreprise proustienne. La vision extérieure, d'une précision et d'une intensité remarquables, de La Comédie humaine tient précisément à ce que le lecteur demeure extérieur au roman. Lisant Balzac, il se fait observateur, moraliste ; bref, il devient un auteur comique qui juge et punit les méfaits et les distractions des personnages. Avec Proust, la lecture devient intérieure et créatrice. Le rapport au roman est aussi bien individuel qu'interactif. La Recherche se conclut par une surprenante ouverture: nous pouvons tous transformer la prétendue banalité de notre vie en quelque chose de grandiose. Il y aura autant de romans qu'il y aura de lecteurs, alors que pour Balzac, un seul grand livre suffit, celui de la Société. Toutefois, cela demande un effort, une intuition dirait l'auteur de L'Évolution créatrice. C'est un effort intérieur devant lequel plusieurs reculent (Swann, Charlus, Saint-Loup sont des êtres sensibles, mais des artistes ratés): «Aussi combien s'en tiennent là qui n'extraient rien de leur impression, vieillissent inutiles et insatisfaits, comme des célibataires de l'art! Ils ont les chagrins qu'ont les vierges et les paresseux, et que la fécondité dans le travail guérirait. Ils sont plus exaltés à propos 
des œuvres d'art que les véritables artistes, car leur exaltation n'étant pas pour eux l'objet d'un dur labeur d'approfondissement, elle se répand au dehors, échauffe leurs conversations, empourpre leur visage» (TR, p. 198). Pour devenir artiste, il faut tordre notre intelligence, notre habitude et notre vanité. La morale de l'œuvre proustienne, tragique parce qu'individuelle et introspective, ne peut être réaliste. Elle est spiritualiste.

\section{Bibliographie}

BALZAC, Honoré de. 1976 [1842], "Avant-propos », dans La Comédie humaine I. Études de mœurs, Paris, Gallimard, coll. « Bibliothèque de la Pléiade ».

—. 2010 [1843], Illusions perdues, Paris, Flammarion, coll. « GF ».

—. 2006 [1847], Splendeurs et misères des courtisanes, Paris, Flammarion, coll. « GF ».

BERGSON, Henri. 2010 [1896], Matière et mémoire. Essai sur la relation du corps à l'esprit, Paris, Presses universitaires de France, coll. «Quadrige (Grands Textes) ».

-. 2007 [1900], Le Rire. Essai sur la signification du comique, Paris, Presses universitaires de France, coll. "Quadrige (Grands Textes) ».

DESCOMBES, Vincent. 2009 [1987], Proust. Philosophie du roman, Paris, Minuit, coll. « Critique ». 
DOUBROVSKY, Serge. 1988 [1982], "Corps du texte/texte du corps », dans Autobiographiques. De Corneille à Sartre, Paris, Presses universitaires de France, coll. "Perspectives critiques $»$.

DUBOIS, Jacques. 2000, Les Romanciers du réel. De Balzac à Simenon, Paris, Seuil, coll. « Points (Essais)».

PROUST, Marcel, À la recherche du temps perdu, édition établie sous la direction de Jean-Yves Tadié.

—. CS. 1988 [1913], Du côté de chez Swann, Paris, Gallimard, coll. « Folio Classique ».

—. JF. 1988 [1919], À l'ombre des jeunes filles en fleurs, Paris, Gallimard, coll. « Folio Classique ».

-. CG. 1988 [1920-1921], Le Côté de Guermantes, Paris, Gallimard, coll. « Folio Classique ».

-. SG. 1989 [1921-1922], Sodome et Gomorrhe, Paris, Gallimard, coll. « Folio Classique ».

—.Pr. 1989 [1925], La Prisonnière, Paris, Gallimard, coll. « Folio Classique ».

—. AD. 1992 [1927], Albertine disparue, Paris, Gallimard, coll. « Folio Classique ».

—. TR. 1990 [1927], Le Temps retrouvé, Paris, Gallimard, coll. «Folio Classique».

—. CSB. 2006 [1954], Contre Sainte-Beuve, Paris, Gallimard, coll. «Folio Essais ». 


\title{
Résumé
}

En analysant la place que prend Honoré de Balzac dans l'œuvre proustienne, cet article souhaite établir une comparaison stylistique entre l'auteur de la Comédie humaine et celui d'À la recherche du temps perdu. Le roman de Marcel Proust est riche des enseignements de l'entreprise balzacienne, ce qui ne veut pas dire qu'il ne tentera pas de la dépasser, au contraire. À l'aide du philosophe Henri Bergson, particulièrement avec son ouvrage Le Rire, sera ainsi expliquée la différence esthétique, voire poétique, entre les écrits de Balzac et ceux de Proust, le second reprenant le grand projet réaliste du premier pour le réfracter dans l'introspection créatrice de son hérosnarrateur, ce qui fait de la Recherche une comédie humaine intérieure.

\begin{abstract}
Considering Honoré de Balzac's place in the works of Marcel Proust, this paper wishes to establish a stylistic comparison between the author of The Human Comedy, and that of In Search of Lost Time (also translated as Remembrance of things past). Proust's novel is full of Balzac's lessons, which, however, does not mean he will not try to surpass Balzac's undertaking, in his own way. Through the philosopher Henri Bergson, especially with his book Laughter, will be explained the aesthetic difference between Balzac's and Proust's writings. Proust is taking up Balzac's major realistic project, but refracting it in the creative introspection of his hero, making In Search of Lost Time an all-personal human comedy.
\end{abstract}

